The column in this issue is supplied by Horacio J. Adrogué, M.D., chief of the Clinical Nephrology Service at Houston Methodist Hospital. Dr. Adrogué is a world-renowned authority on the subject of hyponatremia and has published extensively on this matter, making seminal contributions to the medical literature.

\title{
HYPONATREMIA IN HEART FAILURE
}

\author{
Horacio J. Adrogué, M.D. \\ Houston Methodist Hospital, Houston, Texas
}

Hyponatremia is the most common electrolyte disorder and is frequently encountered in patients with advanced heart failure. Hypotonic or dilutional hyponatremia, usually simply called hyponatremia, is defined as a reduction in the serum sodium concentration to a level below $136 \mathrm{mEq} / \mathrm{L}$. This condition represents an excess of water in relation to existing sodium and potassium stores, since the sodium concentration is determined by Edelman's Equation - the ratio of the "exchangeable" (i.e., osmotically active) portions of the body's sodium and potassium content to total body water. The development of hyponatremia in patients with heart failure increases morbidity and predisposes to falls, hip fractures, and other complications as well as hospital admissions.

At an early stage of congestive heart failure, retention of sodium and water by the kidney causes expansion of extracellular fluid volume and peripheral edema, but not hyponatremia. However, at late-stage congestive heart failure, patients exhibit an impairment in the renal excretion of water (aquaresis or water diuresis), predisposing them to the development of hyponatremia. An increase in the antidiuretic hormone in this late stage imposes an aquaretic defect, which, in combination with the use of potent diuretics and severe salt restriction, frequently leads to dilutional hyponatremia.

The rationale for the development of volume expansion without hyponatremia in heart failure becomes apparent when comparing this condition to the normal state. For example, let us consider a daily oral intake of the following: $\mathrm{Na}^{+}(140 \mathrm{mEq}), \mathrm{K}^{+}(70 \mathrm{mEq})$, and water $(2 \mathrm{~L})$. If we divide the total daily intake of $\mathrm{Na}^{+}$plus $\mathrm{K}^{+}(210$ $\mathrm{mEq})$ by the normal serum sodium concentration $(140 \mathrm{mEq} / \mathrm{L})$, we obtain $1.5 \mathrm{~L}$; this volume is called the "isotonic component" of the intake since it has the same tonicity as plasma. Considering that the water intake is $2 \mathrm{~L}$ and the calculated "isotonic component" is 1.5 L, the difference of $0.5 \mathrm{~L}$ represents "electrolyte-free water." In the normal state, excretion (mostly urine) matches intake so that the balance of the "isotonic component" and the "electrolyte-free water" is zero. Excessive retention of sodium and water by the kidney in early heart failure leads to a positive balance of "isotonic component" that is expressed as extracellular fluid volume expansion and edema. At this early stage, the excretion of "electrolyte-free water" is largely preserved so that its balance remains zero, the same as in the normal state.

Let us now consider how hyponatremia develops in advanced heart failure. In the normal state with a total combined daily $\mathrm{Na}^{+}$ and $\mathrm{K}^{+}$intake of $210 \mathrm{mEq}$ and $2 \mathrm{~L}$ of water, the calculated "electrolyte-free water" intake is $0.5 \mathrm{~L} / \mathrm{d}$. However, salt restriction prescribed by the physician can drastically increase the "electrolyte-free water" intake. If, for example, the intake of $\mathrm{Na}^{+}$plus $\mathrm{K}^{+}$ is $70 \mathrm{mEq} / \mathrm{d}$ and water intake remains at $2 \mathrm{~L} / \mathrm{d}$, the calculated "isotonic component" is now $0.5 \mathrm{~L} / \mathrm{d}$ and the "electrolyte-free water" increases 3-fold, from $0.5 \mathrm{~L} / \mathrm{d}$ in the normal state to $1.5 \mathrm{~L} / \mathrm{d}$. Consequently, unless the patient has a 3-fold increase in the renal excretion of water, dilutional hyponatremia will develop.

Potent diuretics (e.g., loop agents) are prescribed to prevent or correct a positive balance of "isotonic component" that leads to volume expansion. However, the use of potent diuretics decreases the body's stores of $\mathrm{Na}^{+}$and $\mathrm{K}^{+}$- the numerator of the Edelman Equation-therefore predisposing to dilutional hyponatremia. In addition, diuretic-induced volume contraction stimulates thirst (increased water intake) and antidiuretic hormone secretion, thereby promoting water retention and further predisposing to hyponatremia.

Cautious use of diuretics, thoughtful advice on dietary electrolyte intake, and periodic monitoring of serum electrolytes help prevent hypotonic hyponatremia in patients with heart failure. Discontinuing or reducing the dosage of diuretics, lifting the salt restriction, and prescribing a limited fluid intake are the basic tools for managing hyponatremia in patients with heart failure. 